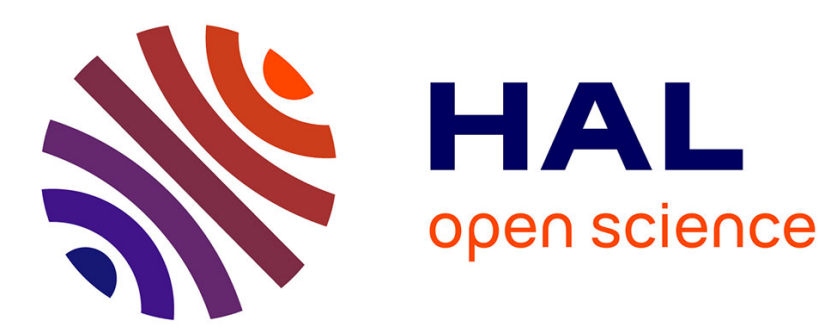

\title{
Production de peroxyde d'hydrogène par le mycélium du champignon de couche et sa contribution à la dégradation des constituants humiques des composts
} Jean-Pierre Goulas

\section{- To cite this version:}

Jean-Pierre Goulas. Production de peroxyde d'hydrogène par le mycélium du champignon de couche et sa contribution à la dégradation des constituants humiques des composts. Agronomie, 1987, 7 (10), pp.853-858. hal-00884961

\section{HAL Id: hal-00884961 https://hal.science/hal-00884961}

Submitted on 1 Jan 1987

HAL is a multi-disciplinary open access archive for the deposit and dissemination of scientific research documents, whether they are published or not. The documents may come from teaching and research institutions in France or abroad, or from public or private research centers.
L'archive ouverte pluridisciplinaire HAL, est destinée au dépôt et à la diffusion de documents scientifiques de niveau recherche, publiés ou non, émanant des établissements d'enseignement et de recherche français ou étrangers, des laboratoires publics ou privés. 


\title{
Production de peroxyde d'hydrogène par le mycé- lium du champignon de couche et sa contribu- tion à la dégradation des constituants humiques des composts
}

\author{
Jean-Pierre GOULAS \\ I.N.R.A., Station de Recherches sur les champignons, Centre de Recherches de Bordeaux, F 33140 Pont-de-la- \\ Maye
}

RESUMÉ Le système catalase/3-aminotriazole se trouve inactivé au contact du mycélium du champignon de couche $A g a$ -
ricus bisporus (Lange) ce qui peut être interprété comme une preuve de la production active de peroxyde
d'hydrogène par le thalle de cryptogame.
A des niveaux de concentrations tout à fait comparables, le peroxyde d'hydrogène en présence d'ions $\mathrm{Cu}$ II
dégrade des produits du type mélanine, assimilables à ceux qui se forment au cours de la préparation des com-
posts destinés à la culture du champignon.
Une représentation du mécanisme d'attaque de ces produits par la combinaison peroxyde d'hydrogène/Cu II
est proposée. Mots clés additionnels : Agaricus bisporus, mélanines, cuivre, décoloration.

Production of hydrogen peroxide by mycelium of the cultivated mushroom and its involvement in decomposition of the humic compounds of composts.

\begin{abstract}
The catalase/aminotriazole system was inactivated in contact with mycelium of the cultivated mushroom Agaricus bisporus (Lange), which may be interpreted as a proof of active production of hydrogen peroxide by the vegetative system of the fungus. At quite comparable concentration levels, hydrogen peroxide in the presence of cupric ions degraded melanin-like products similar to those that arise during the preparation of composts designed for mushroom cultivation. A scheme of the mechanism of attack of the hydrogen peroxide cupric combination on these products is proposed.
\end{abstract}

Additional key words : Agaricus bisporus, melanins, copper, discoloration.

\section{INTRODUCTION}

Un des traits marquants des transformations qui se déroulent au cours de la préparation des composts destinés à la production du champignon de couche se rapporte au processus d'humification qui intervient alors et qui se manifeste notamment par un brunissement de plus en plus prononcé des matériaux soumis à la fermentation.

Les caractéristiques des matières de type humique qui prennent ainsi naissance et leur évolution au cours du travail des fumiers ont déjà fait l'objet d'études détaillées (GRABBE \& HAIDER, 1971; MAGGIONI, 1981).
Une fraction notable du carbone et de l'azote des composts peut être extraite en milieu alcalin et se trouve associée à des produits de couleur foncée qui précipitent par acidification.

On sait que ce processus d'humification va de pair avec le développement d'une certaine stabilisation des constituants organiques du milieu, en particulier des matières azotées.

Il a été montré à plusieurs reprises que l'azote protidique engagé dans des complexes avec des polymères de type humique (ou mélanique) présente une grande résistance à la dégradation par les microorganismes; ce fait s'observe aussi bien dans le cas de substances modèles synthétisées in vitro, que dans celui des matiè- 
res brunes élaborées par certains micromycètes isolés du sol (LADD \& BRISBANE, 1967 ; MARTIN \& HAIDER, 1969 ; VERMA et al., 1975).

On peut se demander dans quelle mesure les matières azotées humifiées du compost restent utilisables par le mycélium du champignon de couche.

C'est un fait d'observation banal que la teinte du milieu se modifie au fur et à mesure que progresse l'envahissement par le cryptogame, passant du brun noir au brun rougeâtre, ce qui amène à penser que les matières brunes sont activement dégradées par cet organisme (MAGGIONI, 1981). L'analyse de l'évolution des acides humiques au cours de la culture paraît contredire toutefois cette interprétation (GRABBE \& HAIDER, 1971).

Dans le cas des composts de champignonnières, on en est encore réduit à des hypothèses quant au mode de formation de ces substances noires et à leur nature exacte. Les investigations de GRABBE \& HAIDER (1971) ont montré que ces produits pourraient dériver en partie de métabolites d'origine microbienne et s'apparenteraient donc à des pigments du type mélanine (RAST et al., 1981 ; HAIDER \& MARTIN, 1970).

Une caractéristique de cette catégorie de substances est la facilité avec laquelle elles sont décomposées par des agents oxydants forts tels que l'eau oxygénée (NiCOlaus et al., 1964), espèce chimique dont l'utilisation pour ses propriétés décolorantes est bien connue (HoLsT, 1954). L'action de $\mathrm{H}_{2} \mathrm{O}_{2}$ peut être considérablement renforcée par l'apport de certains catalyseurs tels que Fe II et s'exerce encore de façon notable à des concentrations très faibles comme cela a été prouvé pour la dégradation de la cellulose (HALLIWELL, 1965). Dans des conditions similaires, l'eau oxygénée oxyde les acides fulviques, ce qui se traduit par une réduction de la teneur en radicaux libres et une diminution de l'absorption en lumière visible (SENESI et al., 1977).

KOENIGS (1972 et 1974) a démontré la production de peroxyde d'hydrogène dans le milieu environnant par un certain nombre de champignons destructeurs du bois.

On s'est proposé dans le présent travail de mettre en évidence l'élaboration de peroxyde d'hydrogène par le champignon de couche Agaricus bisporus (Lange) et de voir si, dans des conditions compatibles avec les exigences biologiques de cet organisme, cet agent d'oxydation pouvait contribuer à la dégradation des substances de type humique ou mélanique présentes dans le milieu.

\section{MATÉRIEL ET MÉTHODES}

\section{A. Mise en évidence de la production d'eau oxygénée par le champignon}

La technique adoptée, empruntée à KoENIGS (1974) après quelques modifications, utilise le fait que la catalase, en présence de 3-aminotriazole, donne avec le peroxyde d'hydrogène un complexe stable, ce qui se manifeste par une inhibition irréversible de l'activité enzymatique. La fraction de la catalase non convertie par $\mathrm{H}_{2} \mathrm{O}_{2}$ ne forme seulement avec l'aminotriazole qu'une combinaison labile et se trouve réactivée par simple dilution. La détermination du pouvoir catalasique résiduel fournit alors une évaluation en retour de la quantité d'eau oxygénée qui est apparue au contact du système catalase/aminotriazole (COHEN \& HoCHSTEIN, 1964).

A cet effet, $35 \mathrm{~g}$ de «blanc de champignon "* (souche 665 Somycel, préculture sur seigle) sont disposés dans des fioles coniques, additionnés de $7,5 \mathrm{ml}$ de la préparation catalase/aminotriazole et sont laissés séjourner pendant le temps requis à une température de $21 \pm 1{ }^{\circ} \mathrm{C}$. A l'issue de cette période, une aliquote de $500 \mu \mathrm{l}$ est prélevée et diluée au $1 / 30^{\mathrm{e}}$ avec de l'eau déionisée. L'activité catalase de la solution est aussitôt analysée selon le procédé décrit par COHEN \& HOCHSTEIN (1964) (réaction avec une préparation 0,006 M d'eau oxygénée en milieu tampon pendant $3 \mathrm{mn}$ à $0^{\circ} \mathrm{C}$ et titrage du substrat non décomposé par le permanganate).

On détermine de façon concomittante :

- le titre Tm correspondant à l'activité maximum exercée par la préparation de catalase initiale à laquelle on a assigné arbitrairement la valeur 100 ;

- le titre To correspondant à l'activité nulle en substituant à l'apport d'enzyme un volume équivalent d'eau déionisée.

La décomposition de l'eau oxygénée par la catalase suit une cinétique du premier ordre qui s'exprime par la relation

$$
K=(1 / \tau) \log \left(a_{0} / a\right)
$$

où $\mathrm{a}=$ concentration au bout du temps $\tau$ et $\mathrm{a}_{0}=$ concentration initiale en $\mathrm{H}_{2} \mathrm{O}_{2}$. Pour une durée de réaction maintenue invariable, la constante de vitesse $\mathrm{K}$ est en relation linéaire avec le logarithme de la concentration résiduelle en $\mathrm{H}_{2} \mathrm{O}_{2}$

$$
\mathbf{K}=-\mathbf{A} \log \mathbf{a}+\mathbf{B} \text {. }
$$

La détermination des coefficients de cette droite à partir de Tm et To fournit la relation qui permet de déduire en fonction du titre $\mathrm{T}$ mesuré, le pourcentage d'activité enzymatique qui subsiste après un certain temps de contact entre la préparation de catalase et le mycélium du champignon.

\section{$\mathrm{K}=-100 \log \mathrm{T} /(\log \mathrm{To}-\log \mathrm{Tm})$}

$+100 \log \mathrm{To} /(\log \mathrm{To}-\log \mathrm{Tm})$.

La production de peroxyde d'hydrogène est alors considérée comme proportionnelle au dégré d'inactivation de la catalase par le système aminotriazole $/ \mathrm{H}_{2} \mathrm{O}_{2}$ émis dans le milieu (KOENIGS, 1972).

\section{Préparation de la solution de catalase/aminotriazole :}

$130 \mu \mathrm{l}$ de la suspension de catalase (EC1.11.1.6 Sigma C 30) correspondant à 138000 unités Sigma sont filtrés sur membrane Millipore EG de $25 \mathrm{~mm}$ de diamètre, laissés essorer, puis entraînés dans une fiole jaugée avec un jet d'eau déionisée. Après addition de $4,2 \mathrm{ml}$ d'une solution à 5 p. 100 de 3-amino 1,2,4-triazole (Aldrich), la solution est complétée à $50 \mathrm{ml}$ avec de l'eau.

\footnotetext{
* Blanc de champignon : culture pure du mycélium de l'Agaric sur un milieu à base de grains de céréales (ou de fumier stérilisé) et qui sert de levain intermédiaire pour l'ensemencement des composts de champignonnières.
} 


\section{B. Action de $\mathrm{H}_{2} \mathrm{O}_{2}$ sur les matières humiques}

Des essais préliminaires ont montré que l'activité décolorante de $\mathrm{H}_{2} \mathrm{O}_{2}$ était encore appréciable à des concentrations de l'ordre de 2 à $6 \cdot 10^{-2} \mathrm{M}$, soit sensiblement inférieures à celle indiquée par HALLIWELL (1965) lors de ses essais de dégradation de la cellulose. Pour illustrer l'importance de cette action, une dilution appropriée d'une préparation de substances humiques modèles a été laissée réagir avec le peroxyde d'hydrogène $(180 \mu$ moles $)$ à la température de $24{ }^{\circ} \mathrm{C}$ en présence de $\mathrm{Cu}$ II $(1,20 \mu$ atome) comme catalyseur, dans un milieu tampon phosphate $0,1 \mathrm{M}, \mathrm{pH}=7,3$, le volume réactionnel final étant fixé à $4,5 \mathrm{ml}$.

L'évolution de la densité optique est relevée toutes les $8 \mathrm{~h}$ en opérant à $480 \mathrm{~nm}$ avec le tampon dilué pris comme référence.

\section{Préparation des substances humiques modèles :}

$500 \mathrm{mg}$ de pyrogallol (environ $4 \mathrm{mmoles} / 45 \mathrm{ml}$ ) ont été laissés s'oxyder à l'air en présence de $300 \mathrm{mg}$ de glycine dans un milieu potassium hydrogenocarbonate $0,2 \mathrm{M}$. Après $72 \mathrm{~h}$ d'agitation, la densité optique (D.O.) de la préparation diluée $(1,2 \mathrm{ml}$ de dilution au $1 / 10^{e} / 4,5 \mathrm{ml}$ ) dans le milieu tampon phosphate $0,1 \mathrm{M}$ $\mathrm{pH}=7,3$ était de l'ordre de 0,80 .

\section{RÉSULTATS}

La figure 1 montre la réduction manifeste de l'activité enzymatique de la préparation catalase/aminotriazole après un temps de contact relativement court avec le mycélium du champignon. Au bout d'une durée d'incubation de seulement $6 \mathrm{~h}$, plus de 97 p. 100 de l'enzyme présent dans la préparation initiale se trouve inactivé.

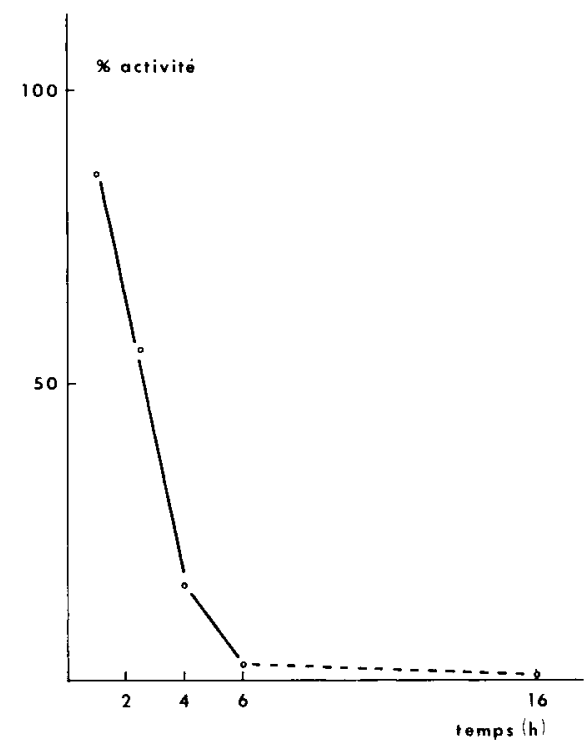

Figure 1

Réduction d'activité du système catalase/3-aminotriazole au contact du mycélium du champignon de couche en fonction du temps. Chaque point représente la moyenne de 2 répétitions.

Loss of activity of the catalase/3-aminotriazole system in contact with mycelium of the cultivated mushroom. Each point represents the average of 2 repeated experiments.
Les faits observés semblent bien constituer une démonstration de l'émission d'eau oxygénée par le mycélium du champignon. D'autres interprétations peuvent toutefois être suggérées. Au cours d'un travail antérieur, il avait été décelé une certaine activité protéase dans le blanc de champignon: de l'ordre de 60 nkatal pour $100 \mathrm{~g}$ de produit $(65 \mathrm{p} .100 \mathrm{de}$ M.S.) avec la caséine comme substrat (souche 56 Somycel). Il s'ensuit que la diminution du pouvoir catalasique peut aussi bien correspondre à une dégradation de l'enzyme par le système protéolytique présent dans le milieu. Pour examiner cette éventualité, on a suivi de façon comparative les modifications du degré d'activité de la catalase, après des périodes d'incubation d'une heure et $4 \mathrm{~h}$ dans 2 groupes d'essais différents : les uns avec l'enzyme seul, les autres avec l'enzyme associé au 3-aminotriazole.

La figure 2 révèle qu'en absence de cette dernière substance, compte tenu de l'écart assez grand entre les résultats obtenus d'une répétition à l'autre, l'activité de la catalase ne semble guère modifiée par le contact avec le thalle du champignon, alors que pour un même intervalle de temps, l'apport d'aminotriazole conduit à une diminution tout à fait démonstrative de la réponse enzymatique.

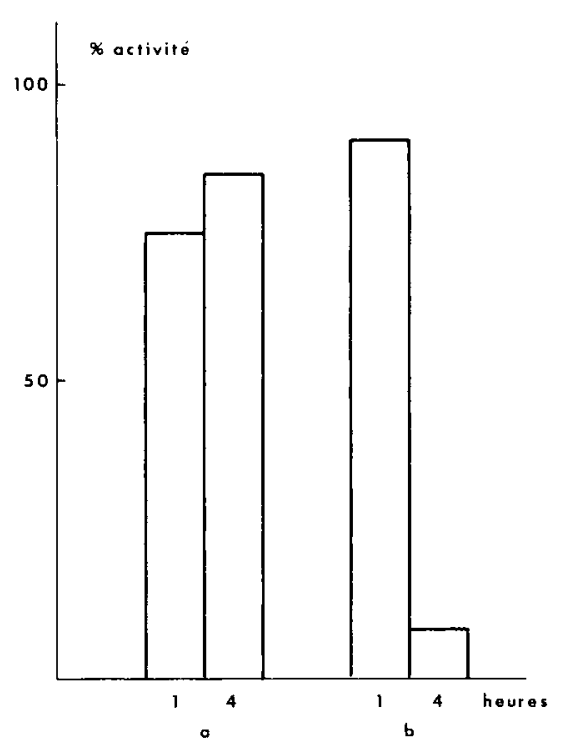

Figure 2

Représentation comparée des modifications d'activité de la catalase a) seule ou b) en présence de 3-aminotriazole après $l$ ou $4 h$ au contact du mycélium du champignon de couche. Chaque barre se rapporte à la moyenne de 2 répétitions.

Comparison of changes in activity of catalase a) alone or $b$ ) in the presence of 3-aminotriazole after 1 or $4 \mathrm{~h}$ in contact with mycelium of the cultivated mushroom. Each bar represents the average of 2 repeated experiments.

L'ensemble de ces résultats paraît donc bien devoir être interprété dans le sens d'une production active d'eau oxygénée par le champignon.

On voit d'après l'évolution des D.O. représentée sur la figure 3 que des concentrations minimes de $\mathrm{H}_{2} \mathrm{O}_{2}$ $\left(410^{-2} \mathrm{M}\right)$ exercent une action tout à fait remarquable sur les composés de type humique. On ignore à quel mode précis de dégradation correspond ce changement des propriétés optiques dans cette région définie du spectre, compte tenu des conditions expérimentales où 


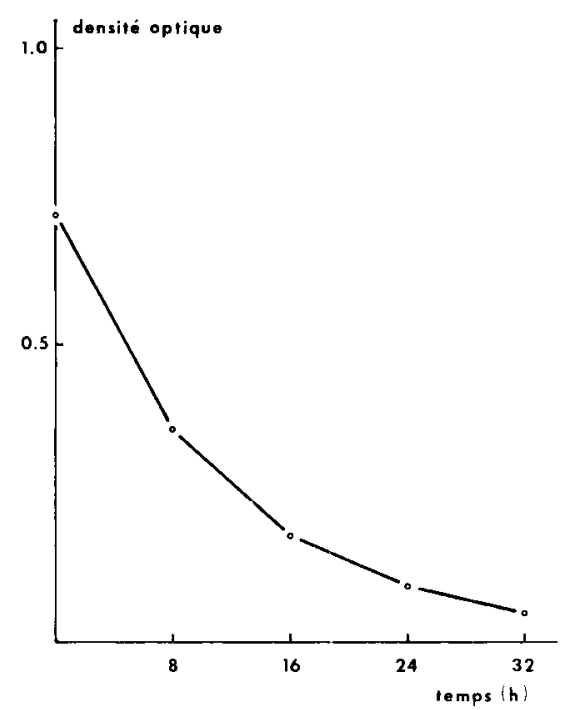

Figure 3

Diminution en fonction du temps de l'absorption à $480 \mathrm{~nm}$ des substances humiques modèles sous l'action du peroxyde d'hydrogène (4. $\left.10^{-2} \mathrm{M}\right)$ en présence du cuivre II.

Decrease with time of the absorbance at $480 \mathrm{~nm}$ of model humic products by action of hydrogen peroxide $\left(4.10^{-2} \mathrm{M}\right)$ in the presence of cupric ions.

l'on s'est placé. A des concentrations plus élevées, l'action du peroxyde d'hydrogène entraîne une destruction profonde du squelette carboné des substances humiques avec libération de produits de faibles masses moléculaires. L'analyse de la courbe obtenue semble indiquer que la diminution de la densité optique suit une cinétique du premier ordre, ce qui incite à penser que la concentration en $\mathrm{H}_{2} \mathrm{O}_{2}$ mise en œuvre est encore en large excès par rapport à celle des groupes chromophores du substrat humique.

\section{TABLEAU 1}

Influence du peroxyde d'hydrogène $\left(4 \cdot 10^{-2} \mathrm{M}\right)$ et/ou du cuivre (1.20 $\mu$ atome) sur l'évolution de l'absorption à $480 \mathrm{~nm}$ des produits humiques modèles pendant 3 jours à $24{ }^{\circ} \mathrm{C}$.

Effect of hydrogen peroxide $\left(4.10^{-2} \mathrm{M}\right)$ and $/$ or copper $(1.20 \mu \mathrm{at}(\mathrm{m})$ on changes in absorbance at $480 \mathrm{~nm}$ of model humic products over a 3 days period at $24{ }^{\circ} \mathrm{C}$

\begin{tabular}{rrrrrr}
\hline \multicolumn{2}{c}{ Additions } & \multicolumn{2}{c}{ Temps O } & \multicolumn{2}{c}{ Temps 3 jours } \\
\hline $\mathrm{H}_{2} \mathrm{O}_{2}$ & $\mathrm{Cu}$ & D.O. & \multicolumn{1}{c}{$\%$} & D.O. & \multicolumn{1}{c}{$\%$} \\
\hline- & - & 0,718 & 100,0 & 0,762 & 106,1 \\
++ & - & 0,714 & 99,4 & 0,451 & 62,8 \\
+ & + & 0,718 & 100,0 & 0,003 & 0,4 \\
- & + & 0,721 & 100,4 & 0,781 & 108,8 \\
\hline
\end{tabular}

Comme l'illustre le tableau 1, l'addition d'ion cuivre se révèle déterminante. L'apport de $1,2 \mu$ mole de sulfate de cuivre conduit à une décoloration complète au bout de 3 jours d'expérience, alors que dans le même temps, en absence de cet élément, l'absorption de la solution ne diminue guère de plus de $40 \mathrm{p}$. 100 . Il n'est d'ailleurs pas exclu que les impuretés introduites à l'état de traces avec les divers produits chimiques intervenant dans l'expérience, puissent exercer alors une certaine action catalytique.

\section{DISCUSSION}

L'activité enzymatique de la combinaison catalase/ 3-aminotriazole est rapidement annihilée sous l'effet d'une culture mycélienne du champignon de couche : Agaricus bisporus (Lange). Il paraît exclu que ce résultat soit le fait de l'action protéolytique du cryptogame. En absence de 3-aminotriazole, l'activité de la catalase introduite dans le milieu se maintient à un niveau élevé, même après $6 \mathrm{~h}$ d'incubation. Il est également peu probable que les faits observés correspondent à un processus d'adsorption de l'enzyme par un quelconque constituant du milieu sur lequel est cultivé le mycélium (grain de seigle stérilisé et divers adjuvants). L'essentiel du processus d'inhibition se développe surtout après la première heure de contact avec le grain, or les phénomènes d'adsorption atteignent généralement leur état d'équilibre beaucoup plus rapidement.

On peut en conclure que l'agaric cultivé possède en commun avec les quelques trente espèces de champignons destructeurs du bois examinés par KOENIGS $(1972,1974)$ la particularité de libérer du peroxyde d'hydrogène de façon active dans le milieu environnant.

Si on se réfère aux données rapportées par KOENIGS (1972), on peut considérer que, dans les conditions expérimentales où s'est placé cet auteur, une production de $150 \mu$ moles d'eau oxygénée entraîne une inhibition d'environ 50 p. 100 du système catalase/aminotriazole. Compte tenu de l'effet de dilution dû à l'humidité du grain, on peut admettre que ces conditions sont très proches de celles réalisées dans la présente étude, en ce qui concerne notamment la concentration en enzyme, soit approximativement 10000 unités Sigma pour $10 \mathrm{ml}$ de milieu.

Entre $1 \mathrm{~h}$ et $4 \mathrm{~h}$ d'incubation, on note un degré d'inactivation de 70 p. 100 de l'activité catalase, ce qui équivaut, si l'on s'en tient aux données ci-dessus, à une émission par le mycélium de l'Agaric d'environ $200 \mu$ moles de peroxyde pour une période de $3 \mathrm{~h}$.

A des niveaux de concentration tout à fait comparables, le peroxyde d'hydrogène en présence de $\mathrm{Cu}$ II exerce une action de dégradation importante sur les produits de type humique ou mélanique.

Les résultats obtenus montrent que ce processus de décomposition reste encore très sensible pour des teneurs en $\mathrm{H}_{2} \mathrm{O}_{2}$ de l'ordre de $20 \mu$ moles $/ \mathrm{ml}$.

Il y a lieu de remarquer que les conditions expérimentales où l'on a opéré s'écartent sans doute notablement de celles qui s'établissent in vivo ; dans ce cas, la production d'eau oxygénée par le mycélium du champignon se trouve alors constamment renouvelée, ce qui en amplifie considérablement l'effet.

L'activité du système ion cuprique/peroxyde d'hydrogène a été reconnue depuis longtemps (BATTIE \& SMEDLEY MC LEAN, 1929) et sa participation à des réactions d'oxydation de types variés a été rapportée à plusieurs reprises (SAUNDERS et al., 1964). Cu II catalyse, entre autres, l'oxydation du catéchol par l'eau oxygénée (INGRAHAM, 1959). L'étude méthodique de cette réaction a permis de montrer que c'est l'ion $[\mathrm{CuOOH}]^{+}$qui semble être l'espèce réagissante. L'ion $[\mathrm{Cu} \mathrm{OOH}]^{+}$représente un oxydant plus actif que le peroxyde d'hydrogène, intervenant aussi bien dans les mécanismes d'oxydation homolytique que dans ceux de type ionique mettant en jeu 2 électrons. Dans 
cette dernière éventualité, l'ion cuprique peut être assimilé à un acide de LEWIS qui en polarisant la liaison entre les atomes d'oxygène du groupement peroxyde, facilite le clivage de cette liaison selon un mode hétérolytique.

Comme c'est le cas pour d'autres substances organiques, la couleur caractéristique des polymères de type humique ou mélanique est en relation avec la présence d'électrons mobiles dont les transitions entre différents états d'énergie correspondent justement à l'adsorption d'un ensemble de radiations dans le domaine visible du spectre. Ces électrons appartiennent à des groupements chromophores constitués par des systèmes de doubles liaisons carbone-carbone et, le cas échéant, carboneoxygène ou carbone-azote conjuguées.

Ces systèmes se retrouvent en particulier dans la configuration moléculaire des substances du type quinone.

Dans le cas d'acides humiques isolés du sol, jusqu'à 70 p. 100 des groupes $C=O$ totaux peuvent être identifiés, à l'analyse, à des fonctions quinones (SCHNITZER \& RIFFALDI, 1972). L'important signal observé en résonnance paramagnétique électronique reste en outre significatif de la présence probable de structures semiquinones dans la composition des groupements fonctionnels associés aux polymères humiques (STEELINK, 1964 ; SCHNITZER et al., 1984).

Les observations de SENESI et al. (1977) laissent supposer que les radicaux libres qui sont en relation avec ces structures sont une composante active des agencements moléculaires constitutifs des groupes chromophores.

On peut penser que l'oxydation de ce type de fonctions représente sans doute la première phase de l'action du système cuivre/eau oxygénée. L'étape déterminante du mécanisme proposé par INGRAHAM (1959) suppose l'oxydation de la semiquinone dérivée du catéchol par l'ion $[\mathrm{Cu} \mathrm{OOH}]^{+}$. Il a déjà été rapporté qu'à faible concentration, $\mathrm{H}_{2} \mathrm{O}_{2}$ oxyde les acides fulviques avec notamment pour effet une diminution du contenu en radicaux libres.

En général, l'augmentation du nombre de doubles liaisons conjuguées s'accompagne d'un déplacement de l'absorption de l'ultra-violet vers les régions visibles du spectre traduisant une diminution de l'énergie de transition des électrons mobiles et, par voie de conséquence, un abaissement relatif du niveau d'énergie des états excités (HOLST, 1954).

Dans des composés complexes tels que les matières humiques, la double liaison constitue donc ainsi un point de moindre résistance dans l'édifice moléculaire et peut représenter aussi le site au niveau duquel va s'effectuer la dégradation par les oxydants (GEISSMAN, 1965).

L'oxydation à l'endroit de la double liaison peut être interprétée comme l'attaque de l'un des doublets électroniques par l'ion $[\mathrm{Cu} \mathrm{OOH}]^{+}$faisant intervenir une réaction d'addition électrophile et amenant la formation transitoire d'un cycle époxyde lié à un proton.

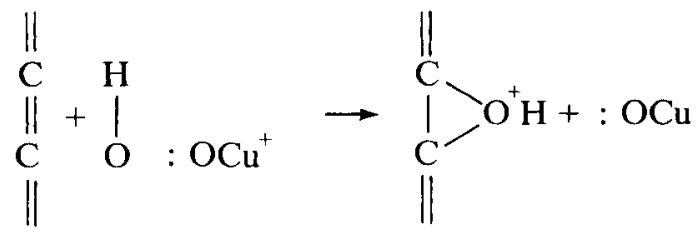

L'ouverture du cycle, avec apparition du glycol, résulte alors, suivant un mécanisme connu, de l'attaque nucléophile par une molécule d'eau à l'arrière de l'un des atomes de carbone (GEISSMAN, 1965).

L'oxydation de l' $\alpha$ diol peut se poursuivre selon le schéma classique aboutissant à la rupture de la liaison carbone-carbone (HOLST, 1954).

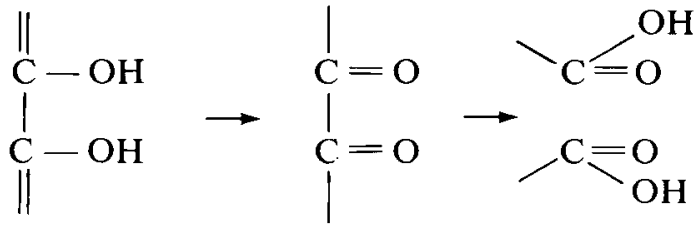

Il est difficile d'apprécier dans quelle mesure in situ, le cuivre à l'état libre ou complexé, peut effectivement prendre part, comme catalyseur, à ce processus d'oxydation.

D'après les analyses de FLECKENSTEIN \& GRABBE (1981), la teneur en cuivre du compost de fumier de cheval au moment de l'ensemencement avec le champignon est de l'ordre de 30 p.p.m. de matière sèche. Environ 1/5 est soluble dans le réactif d'extraction au $\mathrm{CaCl}_{2}$. Rapportée à l'eau d'humidification du compost, cette fraction n'équivaut guère qu'à $0,05 \mu$ atome $\mathrm{de} \mathrm{Cu} / \mathrm{ml}$ de solution. Il est possible qu'à des niveaux de concentration notablement inférieurs à ceux auquels se rapportent les résultats du tableau 1, l'activité du cuivre reste encore appréciable.

Comme c'est le cas pour quelques autres ions métalliques, à l'état libre ou complexé, l'effet catalytique qu'exerce le cuivre peut être assimilé dans une certaine mesure à une activité peroxydase (SAUNDERS et al., 1964).

INGRAHAM (1959) a fait remarquer la similitude qui existe entre l'ion $[\mathrm{Cu} \mathrm{OOH}]^{+}$et l'ion $[\mathrm{Fe} \mathrm{OOH}]^{++}$.

Quelques essais ont montré à l'occasion de la présente étude que le fer pouvait avoir également une action marquée sur la vitesse de décoloration des produits bruns par $\mathrm{H}_{2} \mathrm{O}_{2}$.

C'est le fer ferrique associé à un groupement porphyrine (protoporphyrine IX) qui constitue le cofacteur des enzymes végétales du type peroxydase.

D'après MISHUSTIN \& NIKITIN (1961), la décomposition des humates par les bactéries est due principalement à une activité peroxydase.

Avec le substrat humique particulier sur lequel on a expérimenté, l'addition de peroxydase (EC 1.1.1.7 Sigma type 1) ne modifiait pratiquement pas la marche de la dégradation par l'eau oxygénée. On observait cependant un léger signe d'activité en présence d'un apport complémentaire de résorcinol (cf. SAUNDERS $e t$ al., 1964). L'inhibition de l'enzyme par les matières humiques pourrait expliquer ce défaut apparent d'efficacité (PEREIRA \& MENDEZ, 1976; SCHNITZER et al., 1984). Par ailleurs, les échantillons de blanc sur grains que l'on a examinés pour leurs propriétés enzymatiques paraissaient présenter, comparativement à celle de la laccase, une activité peroxydase très faible, sinon nulle.

La décomposition des acides humiques par les champignons basidiomycètes supérieurs a déjà fait l'objet d'un certain nombre d'expérimentations qui ont montré la réalité du phénomène pour plusieurs espèces lignicoles (BURGES \& LATTER, 1960 ; HURST et al., 
1962 ; TESAŘOVÁ \& SCHÁNĚL, 1968). Les diverses interprétations avancées pour expliciter la biochimie de ce genre d'activité paraissent toutefois quelque peu contradictoires.

Il apparaît maintenant que les modalités de dégradation d'un certain nombre de polymères naturels font intervenir l'action du peroxyde d'hydrogène.

Pour KoENigS (1975), l'association fer/peroxyde d'hydrogène peut être considérée comme équivalente à un système cellulolytique microbien. L'enzyme active dans la dégradation de la lignine que 'TIEN \& KIRK (1984) ont purifié à partir des cultures du basidiomycète Phanerochaete chrysosporium requiert la présence d'eau oxygénée pour exercer son effet.

Pour ce qui est de la décomposition des matières humiques dans le cas particulier des composts de champignonnières, on est à même d'admettre, à lâ suite de la présente étude, qu'il y a également production extracellulaire d'eau oxygénée par le champignon de couche Agaricus bisporus et que ce métabolite peut très bien agir comme agent de dégradation des constituants humiques du milieu dans des conditions compatibles avec la biologie du cryptogame.

On ignore encore cependant si cette action du peroxyde d'hydrogène pourrait être induite par la peroxydase, par un autre type d'enzyme, ou simplement résulter de l'effet catalytique de certains ions métalliques présents dans le compost.

Une telle interprétation de l'action possible du champignon sur les composés humiques se trouve en accord avec les observations rapportées il y a plusieurs décennies par WAKSMAN \& ALLEN (1932), selon lesquelles les constituants organiques du compost, une fois qu'ils ont été soumis, pendant un certain temps, à l'action du mycélium de l'Agaric, sont rendus plus facilement décomposables par la population microbienne du sol.

Ces premiers résultats définissent en partie la nature des rapports sur lesquels se fonde l'adaptation du champignon à son milieu. $\mathrm{Si}$, dans cet ordre d'idée, le rôle essentiel des phénomènes d'humification et de « déshumification » pouvait être confirmé, il y aurait lieu d'en tenir compte dans la mise en œuvre des techniques de préparation des composts en s'efforçant de mieux contrôler l'action des facteurs qui entrent en jeu dans l'élaboration et l'évolution des constituants humiques.

Reçu le 25 septembre 1986. Accepté le 14 juillet 1987.

\section{RÉFÉRENCES BIBLIOGRAPHIQUES}

Battie M. A., Smedley-Mac Lean I., 1929. The catalytic action of cupric salts in promoting the oxidation of fatty acids by hydrogen peroxide. LXVIII. Biochem. J., 23, 593-599.

Burges A., Latter P., 1960. Decomposition of humic acid by fungi. Nature, London, 186, 404-405.

Cohen G., Hochstein P., 1964. Generation of hydrogen peroxide in erythrocytes by hemolytic agents. Biochemistry, 3, 895-900.

Fleckenstein J., Grabbe K., 1981. Quantitative Aufnahms von Schwermetallen aus kontaminierten Substraten des Pilzanbaus durch Agaricus bisporus. Mushr. Sci., XI, Part 2, 35-40.

Geissman T. A., 1965. Principles of organic chemistry. Trad. française J. Colonge, G. Chapas et R. Marey. Dunod, Paris, p. 839.

Grabbe K., Haider K., 1971. Die Huminstoffbildung und der Stickstoffumsatz bei der Bereitung des Kultursubstrats und während des Wachstums von Agaricus bisporus. Z. Pfanzenernähr. Bodenkd., 129, 216-226.

Haider K., Martin J. P., 1970. Humic-acid type phenolic polymers from Aspergillus sydowi culture medium, Stachybotrys spp. cells and autoxidized phenol mixtures. Soil Biol. Biochem., 2, 145-156.

Halliwell G., 1965. Catalytic decomposition of cellulose under biological conditions. Biochem. J., 95, 35-40.

Holst G., 1954. The chemistry of bleaching and oxidizing agents. Chem. Rev., 54, 169-194.

Hurst H. M., Burges A., Latter P., 1962. Some aspects of the biochemistry of humic acid decomposition by fungi. Phytochemistry, 1, $227-231$.

Ingraham L. L., 1959. Model copper oxidases : the cupric ioncatalyzed hydrogen peroxide oxidation of catechol. Arch. Biochem. Biophys, , 81, 309-318.

Koenigs J. W., 1972. Production of extracellular hydrogen peroxide and peroxidase by wood-rotting fungi. Phytopathology, 62, 100-110.

Koenigs J. W., 1974. Production of hydrogen peroxide by woodrotting fungi in wood and its correlation with weight loss, depolyme rization and pH changes. Arch. Microbiol., 99, 129-145.

Koenigs J. W., 1975. Hydrogen peroxide and iron : a microbial cellulolytic system. In Wilke C. R. (Ed.) - Biotechnology and bionei neering Symposium, $\mathrm{n}^{\circ} 5$, Cellulose as a chemical and energy resource. Conference Proceedings Berkeley (Calif. U.S.A.), 25 27 juin 1974, 361 p. John Wiley and Sons : New York/London.

Ladd J. N., Brisbane P. G., 1967. Release of amino acids from soil humic acids by proteolytic enzymes. Aust. J. Soil Res., 5, 161-171.
Maggioni A., 1981. Changes of the humus-like material during composting. Mushr. Sci., XI, Part 2, 47-61.

Martin J. P., Haider K., 1969. Phenolic polymers of Stachybotrys atra, Stachybotrys chartarum and Epicoccum nigrum in relation to humic acid formation. Soil Sci., 107, 260-270.

Mishustin E. N., Nikitin D. I., 1961. (Destructibility of humic acids by soil microflora.) Mikrobiologiya, 30, 841-848 - en russe - In Soils \& Fertilizers XXV (1962), 19 (101).

Nicolaus R. A., Piattelli M., Fattorusso E., 1964. The structure of melanins and melanogenesis. IV. On some natural melanins. Tetrahedron, 20, 1163-1172

Pereira J. R., Méndez J., 1976. Inhibition of peroxidase by algal humic and tulvic acids. Biol. Plant., 18, 179-182.

Rast D. M., Stüssi H., Hegnauer H., Nyhlen L. E., 1981. Melanins. In The fungal spore: morphogenetic controls. G. Turian et M. R. Hohl (Ed.), 507-531. Academic Press., Inc, New York.

Saunders B. C., Holmes-Siedle A. G., Stark B. P., 1964. Peroxidase. Butterworths, London, $281 \mathrm{p}$

Schnitzer M., Riffaldi R., 1972. The determination of quinone groups in humic substances. Soil Sci. Soc. Am. Proc., 36, 772-777.

Schnitzer M., Barr M., Hartenstein R., 1984. Kinetics and characteristics of humic acids produced from simple phenols. Soil Biol. Biochem., 16, 371-376.

Senesi N., Chen Y., Schnitzer M., 1977. The role of free radicals in the oxidation and reduction of fulvic acid. Soil Biol. Biochem., 9, 397-403.

Steelink C., 1964. Free radical studies of lignin, lignin degradation products and soil humid acids. Geochim. cosmochim. Acta, 28, 1615-1622.

Tesařová M., Schánĕl L., 1968. Humus acids as a nutrition source for some white-rot fungi. Biol. Plant., 10, 135-141.

Tien M., Kirk K., 1984. Lignin-degrading enzyme from Phanerochaete chrysosporium : purification, characterization and catalytic properties of a unique $\mathrm{H}_{2} \mathrm{O}_{2}$-requiring oxygenase. Proc. natl. Acad. Sci. USA, 81, 2280-2284.

Verma L., Martin J. P., Haider K., 1975. Decomposition of carbon14-labeled proteins, peptides, and amino acids ; free and complexed with humic polymers. Soil Sci. Soc. Am. Proc., 39, 279-284.

Waksman S. A., Allen M. C., 1932. Comparative rate of decomposition of composted manure and spent mushroom soil. Soil Sci., 34, 189-195. 